\title{
Multi-functionalized carbon nanotubes polymer composites: Properties and applications
}

\begin{abstract}
Carbon nanotubes (CNTs) is a rigid rod-like nanoscale material produced from carbon in powder, liquid, or gel form via acid or chemical hydrolysis. Due to its unique and exceptional renewability, biodegradability, mechanical, physicochemical properties, and abundance, the incorporation associated with a small quantity of CNTs to polymeric matrices enhance the mechanical and thermal resistance, and also stability of the latter by several orders of magnitude. Moreover, NCC-derived carbon materials are of no serious threat to the environment, providing further impetus for the development and applications of this green and renewable biomaterial for lightweight and degradable composites. Surface functionalization of CNTs remains the focus of CNTs research in tailoring its properties for dispersion in hydrophilic and hydrophobic media. Through functionalization, the attachment of appropriate chemical functionalities between conjugated $\mathrm{sp}^{2}$ of CNTs and polymeric matrix is established. It is thus of utmost importance that the tools and protocols for imaging CNTs in a complex matrix and quantify its reinforcement, antimicrobial, stability, hydrophilicity, and biodegradability are be developed.
\end{abstract}

Keyword: CNTs; Composites; Polymer; Functionalization and applications 